\title{
Images of the month 2: A rare case of haematemesis from a gastric submucosal lesion
}

\author{
Authors: Ahmed Hashim, ${ }^{A}$ Zainab Afzal, ${ }^{B}$ Suzanne Ryan ${ }^{C}$ and Patrick Dubois ${ }^{D}$
}

Fig 1. Endoscopic appearance of the large submucosal lesion in the fundus of the stomach. The lesion appears ulcerated with active bleeding.

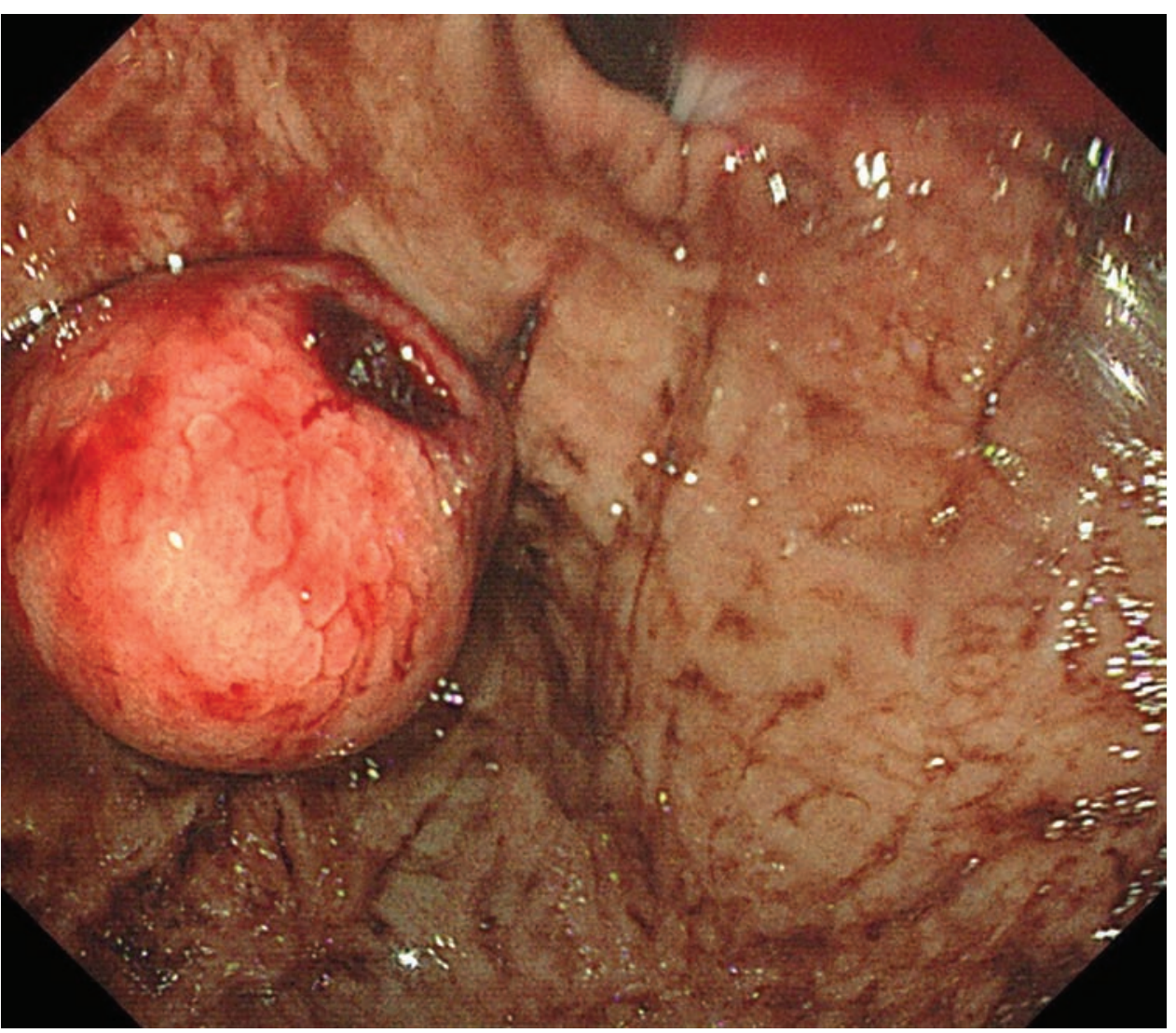

KEYWORDS: Gastric lipoma, haematemesis, gastric liposarcoma

\section{Case presentation}

A 93-year-old male presented to the emergency department with haematemesis along with symptoms of lethargy and decreased

Authors: ${ }^{\mathrm{A}}$ gastroenterology specialist registrar, King's College Hospital, London, UK; B junior clinical fellow, King's College Hospital, London, UK; ' Consultant radiologist, King's College Hospital, London, UK;

${ }^{D}$ consultant gastroenterologist, King's College Hospital, London, UK appetite. Following fluid resuscitation, an upper gastrointestinal endoscopy was performed. A large submucosal lesion with ulceration and oozing blood was observed in the gastric fundus (Fig 1). Endoscopic haemostasis was achieved through injection of adrenaline and application of Haemospray ${ }^{\mathrm{TM}}$ but clips were difficult to place given the size of the ulcer. An initial provisional diagnosis of a gastrointestinal stromal tumour (GIST) was made given the submucosal lesion and bleeding nature of the mass. However, computed tomography (CT) showed a fungating mass arising from the fundus of the stomach, which was of fat density (Fig 2). The patient was conservatively treated, edoxaban was stopped and no further interventions were planned. He had no recurrence of bleeding and was discharged safely. 


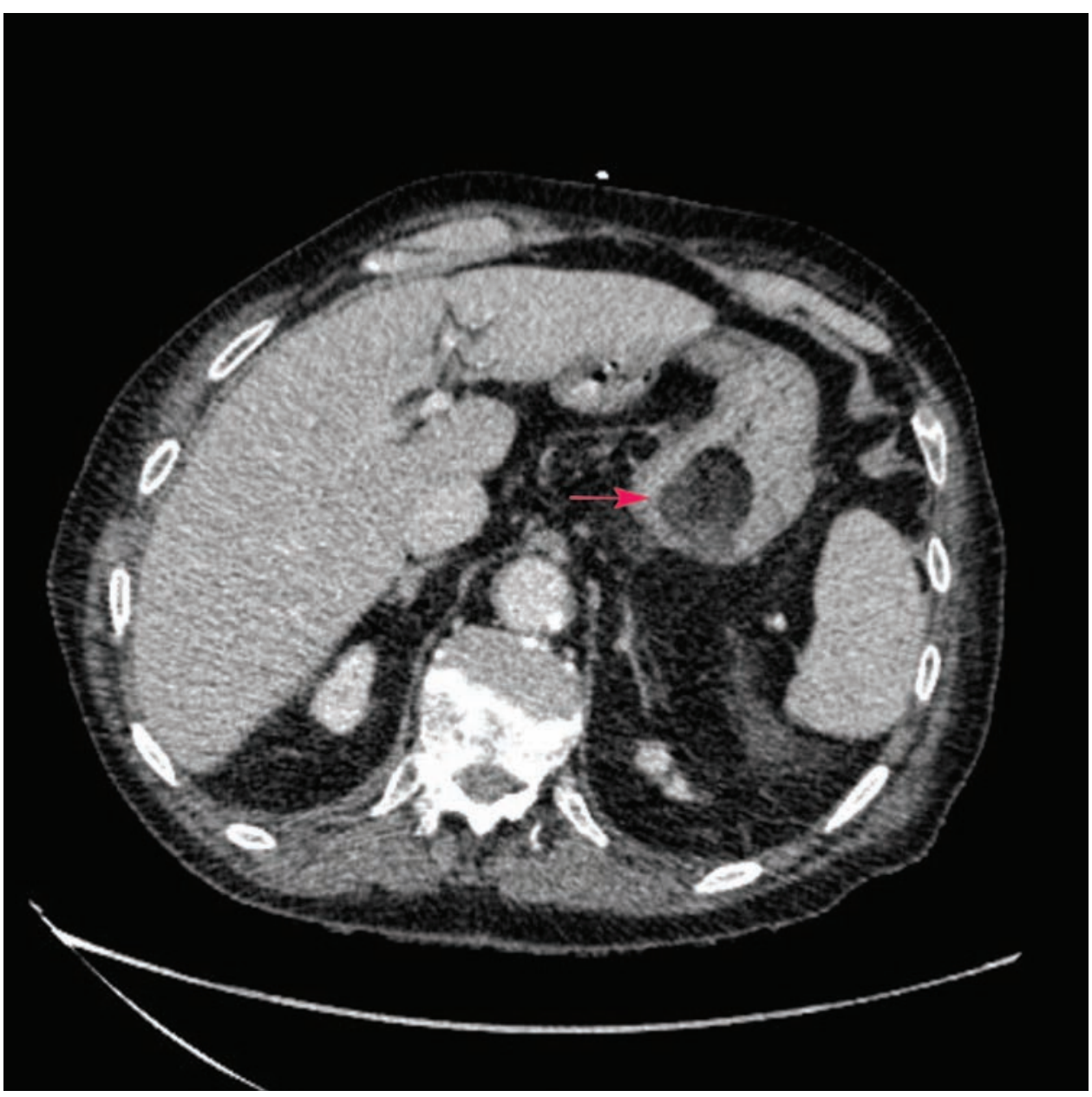

Fig 2. Computed tomography showing the large fungating mass in the fundus of the stomach (arrow) with ulceration which displays low attenuation in keeping with a fat density lesion.

\section{Discussion}

The differential diagnosis in this case included mesenchymal tumours of the stomach such as GIST. However, the CT demonstrated fat density within the lesion, with a negative Hounsfield unit (HU). This makes the lesion most likely to represent either a lipoma or a liposarcoma. Gastric lipomas are rare gastric lesions, accounting for no more than $1 \%$ of gastric tumours and are usually benign in nature. ${ }^{1}$ Most gastric lipomas are asymptomatic and often found incidentally on CT or during endoscopy. CT allows confident diagnosis of a benign lipoma showing low attenuation, fat containing homogeneous appearance which measures between -80 to $-120 \mathrm{HU}$. The presence of septations or marginal defects may indicate ulceration or very rarely a liposarcoma. ${ }^{2}$ Larger lesions may present with dyspepsia symptoms, gastric outlet obstruction or bleeding. ${ }^{2}$ The latter is rare and only a few cases have been reported to date. ${ }^{1-3}$ Our case illustrates rare bleeding from a large gastric lipomatous lesion due to extensive ulceration into the mucosa. The bleeding might have been aggravated by the use of the directly acting anticoagulant (edoxaban).
Management of gastric lipomas is not well described; the most common treatment modalities include partial gastrectomy, endoscopic resection or open enucleation. ${ }^{3}$ Since this patient was an elderly man with multiple co-morbidities, a more conservative approach was taken, and no resection of the mass was planned.

\section{References}

1 Ramdass M], Mathur S, Seetahal-Maraj P, Barrow S. Gastric lipoma presenting with massive upper gastrointestinal bleeding. Case Rep Emerg Med 2013;2013:506101.

2 Amundson JR, Straus D, Azab B et al. Giant symptomatic gastric lipoma: A case report and literature review. Int J Surg Case Rep 2018;51:313-7.

3 Termos S, Reslan O, Alqabandi O et al. Giant gastric lipoma presenting as GI bleed: Enucleation or Resection? Int J Surg Case Rep 2017:41:39-42.

Address for correspondence: Dr Ahmed Hashim, Department of Gastroenterology, King's College Hospital, London SE5 9RS, UK. Email: ahmedsir37@hotmail.com 